PROCEEDINGS OF THE

AMERICAN MATHEMATICAL SOCIETY

Volume 138, Number 12, December 2010, Pages 4281-4289

S 0002-9939(2010)10403-3

Article electronically published on May 17, 2010

\title{
ON CORSON COMPACTA AND EMBEDDINGS OF $C(K)$ SPACES
}

\author{
WITOLD MARCISZEWSKI AND GRZEGORZ PLEBANEK
}

(Communicated by Nigel J. Kalton)

\begin{abstract}
We investigate properties of those compact spaces $K$ for which the Banach space $C(K)$ can be isomorphically embedded into a space $C(L)$, where $L$ is Corson compact. We show that in such a case $K$ must be Corson compact provided $K$ has some additional measure-theoretic property. The result is applicable to Rosenthal compacta and several other classes of compact spaces $K$.
\end{abstract}

\section{Corson compacta and $C(K)$ SPACES}

A compact space $K$ is Corson compact if, for some cardinal number $\kappa, K$ is homeomorphic to a subset of the $\Sigma$-product of real lines

$$
\Sigma\left(\mathbb{R}^{\kappa}\right)=\left\{x \in \mathbb{R}^{\kappa}:\left|\left\{\alpha: x_{\alpha} \neq 0\right\}\right| \leq \omega\right\} .
$$

The class of Corson compacta has been intensively studied for its interesting topological properties and various connections to functional analysis; we refer the reader to a basic paper 1 by Argyros, Mercourakis and Negrepontis, and to extensive surveys by Negrepontis [19] and Kalenda [12. In the sequel, we shall denote the class of Corson compacta by $\mathfrak{C}$. Clearly, $\mathfrak{C}$ is stable under compact subspaces; the class $\mathfrak{C}$ is also stable under continuous images (see e.g. [19, 6.26).

For a compact space $K$ we denote by $C(K)$ the Banach space of real-valued continuous functions with the usual supremum norm. The dual space $C(K)^{*}$ is identified with $M(K)$, the space of all signed Radon measures of finite variation; likewise, $M_{1}(K)$ is the unit ball of $M(K)$, and $P(K)$ denotes the space of Radon probability measures on $K$. Thus every $\mu \in P(K)$ is treated as an inner regular measure defined on the Borel $\sigma$-algebra $\operatorname{Bor}(K)$ of $K$. In the sequel, $M_{1}(K)$ and $P(K)$ are often treated as topological spaces, equipped with the weak* topology inherited from $C(K)^{*}$. Note that we usually write $\mu(g)$ for $\int_{K} g \mathrm{~d} \mu$. For any $x \in K$ we write $\delta_{x} \in P(K)$ for the corresponding Dirac measure; recall that $\Delta(K)=\left\{\delta_{x}: x \in K\right\}$ is a subspace of $P(K)$ which is homeomorphic to $K$.

Suppose that $L$ is Corson compact and $K$ is another compact space such that $C(K) \simeq C(L)$; i.e. they are isomorphic as Banach spaces. Must $K$ be Corson compact? This question has been around for several years; see e.g. 3.9 in [1] 6.45

Received by the editors September 11, 2009 and, in revised form, January 24, 2010.

2010 Mathematics Subject Classification. Primary 46B26, 46E15; Secondary 46E27.

Research of the first author was partially supported by MNiSW Grant No. N N201 382034.

The second author was partially supported by grant 2191/W/IM/09 from the University of Wrocław. 
in Negrepontis [19] or Question 1 in Koszmider [13. We shall consider the following more general question.

Problem 1.1. Let $L$ be Corson compact and suppose that for a compact space $K$ there is an isomorphic embedding $C(K) \hookrightarrow C(L)$. Must $K$ be Corson compact?

We shall denote by $\mathfrak{C}^{*}$ the class of those compact spaces $K$ for which there is an embedding $C(K) \hookrightarrow C(L)$ for some $L \in \mathfrak{C}$; with that convention, Problem 1.1 amounts to asking whether $\mathfrak{C}^{*}=\mathfrak{C}$. It is known that the answer 'yes' is consistent; the result given below is due to Argyros et al. 11.

Theorem 1.2. Let $L$ be a Corson compact space $L$; the following are equivalent:

(i) every $\mu \in P(L)$ has a separable support;

(ii) $M_{1}(L)$ is Corson compact.

Consequently, Martin's axiom $M A\left(\omega_{1}\right)$ implies that $\mathfrak{C}^{*}=\mathfrak{C}$.

There is a long list of Corson compact spaces $K$ constructed under $\mathrm{CH}$ (or weaker axioms) for which $P(K)$ (and $M_{1}(K)$ ) is not Corson compact; see e.g. Haydon [1], Kunen [14, Argyros et al. 11, Kunen and van Mill [15, Plebanek [21, 23]. Nonetheless, the question if Problem 1.1 can be resolved in ZFC remains open.

We note at this point that a list of compacta which plainly do not belong to $\mathfrak{C}^{*}$ is rather short; let us name two such compacta and explain briefly why they are outside $\mathfrak{C}^{*}$; we refer the reader to Talagrand [25] and Plebanek 20] for the terminology and facts that are in use here. If $L \in \mathfrak{C}$, then the Banach space $C(L)$ is realcompact (in its weak topology); in fact $C(L)$ has a stronger property, called the Mazur property ([20]). Both realcompactness and the Mazur property are preserved by taking closed linear subspaces. The space $\left[0, \omega_{1}\right]$, of ordinals $\leq \omega_{1}$ with the topology induced by order, is not in $\mathfrak{C}^{*}$ because $C\left[0, \omega_{1}\right]$ is not realcompact. Accordingly, $\beta \omega \notin \mathfrak{C}^{*}$ as $C(\beta \omega)$ does not have the Mazur property. Arguments of this type have been recently used by Galego 8 to prove some structure results on the spaces of continuous functions on dyadic compacta.

The split interval is another classical compactum which is not Corson compact (since it is separable but not metrizable). In fact the split interval does not belong to $\mathfrak{C}^{*}$, as will become clear in the sequel, as a corollary to the following result.

Theorem 1.3. If $K \in \mathfrak{C}^{*} \backslash \mathfrak{C}$, then the compact space $M_{1}(K)$ carries a regular measure, the support of which is not separable.

Proof. If $T: C(K) \rightarrow C(L)$ is an isomorphic embedding for some $L \in \mathfrak{C}$, then we can as well assume that $T[C(K)]$ separates the points of $L$. Indeed, define an equivalence relation $\sim$ on $L$ by saying that

$$
t \sim s \text { if } T(f)(t)=T(f)(s) \text { for every } f \in C(K) .
$$

Let $L^{\prime}$ be the space of equivalence classes of $\sim$ and let $q: L \rightarrow L^{\prime}$ be the quotient map. Then $L^{\prime}=q[L]$ is Corson compact, and it suffices to notice that $C(K)$ embeds into $C\left(L^{\prime}\right)$. But this follows from the fact that if $g \in C(L)$ is constant on equivalence classes of $\sim$, then $g=g^{\prime} \circ q$ for some $g^{\prime} \in C\left(L^{\prime}\right)$.

Now, consider the conjugate map

$$
T^{*}: M(L) \rightarrow M(K), \quad T^{*}(\nu)(f)=\nu(T f) .
$$

Since $T$ is an isomorphic embedding, then $T^{*}$ is surjective, and it follows that $M_{1}(K) \subseteq c \cdot T^{*}\left[M_{1}(L)\right]$ for some constant $c>0$. As $K$ is not Corson, the space 
$M_{1}(K)$, containing $K$ topologically, is not Corson either; consequently, $M_{1}(L)$ is not Corson compact.

By Theorem 1.2 there is a measure $\nu \in P(L)$ the support of which is not separable. Note that $T^{*}$ is injective on $\Delta(L)=\left\{\delta_{l}: l \in L\right\}$ since $T[C(K)]$ separates the points of $L$. Hence $T^{*}$ defines a homeomorphic embedding of $L \cong \Delta(L)$ into $r M_{1}(K)$ for some $r>0$. It follows that the space $M_{1}(K)$ admits a regular measure which does not have separable support.

Following [1] we say that a space $K$ has property $(M)$ if the support of every $\mu \in P(K)$ is separable. Note that, however, here we mean 'the' support, i.e. the smallest closed set of full measure (not just the fact that $\mu$ is concentrated on a separable subspace; see the remark at the end of section 3 ). In view of Theorem 1.3 it is worth investigating for which compact spaces $K$ the space $M_{1}(K)$ has property $(M)$.

Definition 1.4. Let us say that a compact space $K$ has property $\left(M^{*}\right)$ if the support of every measure $\mathbf{v} \in P\left(M_{1}(K)\right)$ is separable.

Using this terminology we can rephrase Theorem 1.3 as follows.

Corollary 1.5. If $K$ has property $\left(M^{*}\right)$ and $K \in \mathfrak{C}^{*}$, then $K \in \mathfrak{C}$.

\section{Countably Determined measures and property $\left(M^{*}\right)$}

The property $\left(M^{*}\right)$ introduced in the previous section may be investigated with the help of the following concept from topological measure theory. A measure $\mu \in P(K)$ is said to be countably determined if there is a countable family $\mathcal{F}$ of closed sets such that

$$
\mu(U)=\sup \{\mu(F): F \subseteq U, F \in \mathcal{F}\},
$$

for every open $U \subseteq K$; see Pol [24] and Mercourakis [18]. It will be convenient to say, whenever $(\dagger)$ holds, that $\mathcal{F}$ approximates $U$ from below (with respect to some $\mu \in P(K)$, which is clear from the context).

We shall now recall several standard properties of countably determined measures.

Lemma 2.1. Let $K$ be any compact space and $\mu \in P(K)$.

(a) If there is a countable family $\mathcal{B}$ of Borel sets approximating all open sets from below, then $\mu$ is countably determined.

(b) If a countable family $\mathcal{B} \subseteq B$ or $(K)$ approximates from below each element of some pseudobase $\mathcal{H}$ of the topology on $K$, then $\mu$ is countably determined.

(c) If $\mu$ is countably determined, then the support of $\mu$ is separable.

Moreover, the class of compact spaces on which every regular measure is countably determined is finitely productive and closed under continuous images.

Proof. (a) follows immediately by regularity of $\mu$. In (b) we note that the smallest lattice $\mathcal{L}$ generated by $\mathcal{B}$ is countable and that $\mathcal{L}$ approximates from below the lattice $\mathcal{U}$ generated by $\mathcal{H}$, where $\mathcal{U}$ is a base closed under finite unions. By $\tau-$ additivity of Radon measures, $\mathcal{U}$ approximates all open sets from below.

To check (c) we take a countable family $\mathcal{F}$ of closed sets of positive measure witnessing that $\mu$ is countably determined. Let $S \subseteq K$ be the support of $\mu$; then $F \cap S \neq \emptyset$ for $F \in \mathcal{F}$ and if we pick $s_{F} \in F \cap S$ for every $F \in \mathcal{F}$, then $S$ is the closure of $\left\{s_{F}: F \in \mathcal{F}\right\}$. 
The second part can be checked in a standard way; see Pol 24, Lemma 3.6.

Now we have come to the main point of the section.

Theorem 2.2. Suppose that every $\mu \in P(K)$ is countably determined. Then $K$ has property $\left(M^{*}\right)$.

The theorem follows directly from Lemma 2.1(c) and the following lemma, which is due to Pol 24], Lemma 4.4; we enclose a (slightly different) proof of the latter.

Lemma 2.3. If every $\mu \in P(K)$ is countably determined, then every measure $\mathbf{v} \in P\left(M_{1}(K)\right)$ is again countably determined.

Proof. (1) We consider first measures $\mathbf{v} \in P(P(K))$. In the sequel, $\mathbf{x} \in P(K)$ always denotes a variable in the formulae.

For every $g \in C(K)$ we write $\varphi_{g}: P(K) \rightarrow \mathbb{R}$ for the continuous function given by $\varphi_{g}(\mathbf{x})=\mathbf{x}(g)=\int_{K} g \mathrm{~d} \mathbf{x}$, for $\mathbf{x} \in P(K)$. We also consider mappings $\varphi_{h}$ for $h: K \rightarrow \mathbb{R}$ which are not necessarily continuous. Observe that for any closed $F \subseteq K, \varphi_{\chi_{F}}$ is upper semicontinuous.

Every regular measure $\mathbf{v}$ on $P(K)$ has a barycenter since $P(K)$ is compact and convex; see e.g. Diestel [4], Chapter IX. This (in particular) means that there is $\mu \in P(K)$ such that for any $g \in C(K)$ we have

$$
\varphi_{g}(\mu)=\mu(g)=\int_{P(K)} \mathbf{x}(g) \mathrm{d} \mathbf{v}(\mathbf{x}) .
$$

If $F \subseteq K$ is closed, then there is a downward directed family $\left\{g_{a}: a \in A\right\} \subseteq C(K)$, such that $\chi_{F}=\inf _{a \in A} g_{a}$; from this and $(*)$ one can conclude that

$$
\mu(F)=\int_{P(K)} \mathbf{x}(F) \mathrm{d} \mathbf{v}(\mathbf{x})
$$

In turn $(* *)$ shows that the formula $(*)$ is valid for every $\varphi=\varphi_{h}$, where $h$ is a linear combination of $\chi_{F}$, with $F$ closed.

(2) Now fix $\mathbf{v} \in P(P(K))$, and let $\mu$ be its barycenter. By our assumption, $\mu$ is countably determined, so there is a countable family $\mathcal{F}$ of closed sets in $K$ that approximates all open sets from below. The family of the sets

$$
H(g, r)=\{\mathbf{x} \in P(K): \mathbf{x}(g)>r\} \text {, where } g \in C(K), g: K \rightarrow[0,1], r \in \mathbb{R},
$$

is a pseudobase of the weak* topology of $P(K)$. By Lemma 2.1 it is sufficient to prove that the countable family consisting of sets

$$
H(h, r), \text { where } h \in s(\mathcal{H})=\left\{(1 / n) \sum_{i \leq n} \chi_{F_{i}}: n \in \omega, F_{i} \in \mathcal{F}\right\}
$$

approximates that pseudobase from below.

(3) Claim. Let $g: K \rightarrow[0,1]$ be a continuous function. For every $\varepsilon>0$ there is $h \in s(\mathcal{F})$ such that $0 \leq h \leq g$ and $\mathbf{v}\left\{\mathbf{x} \in P(K): \varphi_{g-h}(\mathbf{x})>\varepsilon\right\}<\varepsilon$.

To prove the claim take $n$ so that $1 / n<\varepsilon^{2} / 2$; for every $i<n$ write $V_{i}=\{t \in$ $K: g(t)>i / n\}$ and choose $F_{i} \in \mathcal{F}$ so that $F_{i} \subseteq V_{i}$ and $\sum_{i<n} \mu\left(V_{i} \backslash F_{i}\right)<\varepsilon^{2} / 2$. We have

$$
h=\sum_{i<n} \chi_{F_{i}} \leq \sum_{i<n} \chi_{V_{i}}<g<\sum_{i<n} \chi_{V_{i}}+1 / n,
$$


and we shall check that $h$ is as required. But the inequalities above yield that $\mu(g-h)<\varepsilon^{2}$, so

$$
\int_{P(K)} \varphi_{g-h} \mathrm{~d} \mathbf{v}=\mu(g-h)<\varepsilon^{2},
$$

and the claim follows from the Chebyshev inequality.

(4) Consider now $H=H(g, r)$. Then

$$
B=\bigcup_{h \in s(\mathcal{H}), h<g} H(h, r) \subseteq H(g, r) ;
$$

we shall check that $\mathbf{v}(H(g, r) \backslash B)=0$.

Fix $\delta>0$ and $r^{\prime}$ such that $r+\delta>r^{\prime}>r$ and $\mathbf{v}\left(H(g, r) \backslash H\left(g, r^{\prime}\right)\right)<\delta$. Let $h$ be as in (3) with $\varepsilon=r^{\prime}-r$; thus for a set $E=\left\{\mathbf{x} \in P(K): \varphi_{g-h}>\varepsilon\right\}$ we have $\mathbf{v}(E)<\varepsilon$, and writing $H=H(g, r), H^{\prime}=\left(g, r^{\prime}\right)$, we have

$$
\mathbf{v}(H)-\delta \leq \mathbf{v}\left(H^{\prime}\right) \leq \varepsilon+\mathbf{v}\left(H^{\prime} \backslash E\right) \leq \varepsilon+\mathbf{v}(H(h, r)),
$$

where we used the inclusion $H^{\prime} \backslash E \subseteq H(h, r)$. This verifies the last statement from (2) and completes the proof that the measure $\mathbf{v}$ is countably determined.

(5) Finally, $M_{1}(K)$ is the image of $T \times P(K) \times P(K)$ under the mapping $l(s, t, \mu, \nu)=s \mu-t \nu$, where $T=\left\{(s, t) \in[0,1]^{2}: s+t \leq 1\right\}$. The property 'every measure is countably determined' is finitely productive and closed under continuous images, so the proof is complete.

Every countably determined measure $\mu$ is in particular of countable Maharam type (i.e. $L_{1}(\mu)$ is separable). Let us remark that using part (1) of the proof of Lemma 2.3 one can get the following analogous result.

Proposition 2.4. Suppose that every $\mu \in P(K)$ is of countable type. Then every $\mathbf{v} \in P\left(M_{1}(K)\right)$ is of countable type.

Proof. We again consider some $\mathbf{v} \in P(P(K))$ and its barycenter $\mu \in P(K)$, and we follow the notation of part (1) of the proof of Lemma 2.3. For any $g, h \in C(K)$ and $\mathbf{x} \in P(K)$ we have $|\mathbf{x}(g)-\mathbf{x}(h)| \leq \mathbf{x}(|g-h|)$, and by the barycentric formula $(*)$ this yields

$$
\int_{P(K)}\left|\varphi_{g}-\varphi_{h}\right| \mathrm{d} \mathbf{v} \leq \int_{P(K)} \varphi_{|g-h|} \mathrm{d} \mathbf{v}=\mu(|g-h|) .
$$

Since $\mu$ is of countable type, the above inequality implies that the family

$$
\Phi=\left\{\varphi_{g}: g \in C(K)\right\} \subseteq C(P(K))
$$

is separable in the $L_{1}-$ norm of $L_{1}(\mathbf{v})$. Let $\Psi$ be the subring of $C(P(K))$ generated by $\Phi$; it is routine to check that $\Psi$ is separable too. But $\Psi$ is norm dense in $C(P(K))$ by the Stone-Weierstrass theorem, so $C(P(K))$ is separable in the $L_{1}-$ norm of $L_{1}(\mathbf{v})$. Consequently, $L_{1}(\mathbf{v})$ is itself separable since $C(P(K))$ is its dense subspace.

For the general case we can argue as in (5) of the proof of Lemma 2.3. since the class of compact spaces on which every Radon measure is of countable type is preserved by continuous images and countable products. 


\section{Applichtions AND REMARKS}

We can now present some applications of our considerations.

Corollary 3.1. If $K \in \mathfrak{C}^{*}$, then $K$ is Corson compact under any of the following assumptions:

(i) $K$ is scattered; or

(ii) $K$ is a linearly ordered topological space; or

(iii) $K$ is zero-dimensional and the algebra Clopen $(K)$ is minimally generated in the sense of Koppelberg.

Corollary 3.1 follows immediately from Corollary 1.5 and Theorem 2.2, as any of (i)-(iii) implies that all $\mu \in P(K)$ are countably determined. This is clear in case (i), since every $\mu \in P(K)$ on a scattered compactum is purely atomic (concentrated on a countable set). If $K$ is linearly ordered, then every $\mu$ is again countably determined by a result to be found in $[18$.

For (iii) recall that a Boolean algebra $\mathfrak{A}$ is minimally generated if for some $\kappa$ we can express $\mathfrak{A}$ as $\bigcup_{\xi<\kappa} \mathfrak{A}_{\xi}$, where $\mathfrak{A}_{\xi}$ form a continuous increasing chain of algebras and for every $\xi<\kappa, \mathfrak{A}_{\xi+1}$ is a minimal extension of $\mathfrak{A}_{\xi}$; that is, there is no intermediate algebra $\mathfrak{B}$ between $\mathfrak{A}_{\xi}$ and $\mathfrak{A}_{\xi+1}$. We refer to Borodulin-Nadzieja [2] and [6] for more details. In fact we can extend (iii) beyond zero-dimensional spaces, considering the compact space obtained by inverse limits of transfinite sequences of simple extensions; see [ 6] for further references.

It is now clear that if $K$ is the split interval, then $K \notin \mathfrak{C}^{*}$; we can use either (ii) or (iii) of Corollary 3.1 in this case. Moreover, $K$ is the subject of the result given below. Recall that $K$ is Rosenthal compact if $K$ can be represented as the compact sets of Baire one functions on some Polish space; see [3, [9], [16], 26].

Corollary 3.2. If $K$ is Rosenthal compact and $K \in \mathfrak{C}^{*}$, then $K \in \mathfrak{C}$.

Proof. The point is that by results due to Godefroy [9], if $K$ is Rosenthal compact, then $M_{1}(K)$ is also Rosenthal compact and the support of every regular measure on a Rosenthal compactum is separable. Consequently, every Rosenthal compactum has property $\left(M^{*}\right)$, and we may apply Theorem 2.2 alone here.

Let us mention in connection with Corollary 3.2 that the problem if every measure on a Rosenthal compact space is countably determined seems to be more delicate; see Marciszewski and Plebanek [17. We also note that consistently, under some assumption contradicting $\mathrm{MA}\left(\omega_{1}\right)$, if $K$ is first-countable, then every $\mu \in P(K)$ is countably determined (in fact has some stronger property); see Plebanek 22. It follows that in some models of ZFC in which MA $\left(\omega_{1}\right)$ fails, if $K$ is first-countable and $K \in \mathfrak{C}^{*}$, then $K \in \mathfrak{C}$.

We finish the section by a side remark: Under $\mathrm{CH}$ there is a nonseparable Corson compact $L$ without property $(M)$, such that nonetheless every $\nu \in P(L)$ is of countable type; see Kunen [14. That construction gives a space $L$ and a measure $\mu \in P(L)$ such that $L$ is the support of $\mu$. It follows that $P(L)$ is separable (see e.g. [7]), so when we transfer $\mu$ to a measure $\mathbf{v}$ on $P(L)$ via the mapping $L \ni t \rightarrow \delta_{t} \in P(L)$, the support of $\mathbf{v}$ is not separable though $\mathbf{v}$ lives on a separable space $P(L)$. 


\section{USING CALIBERS}

We do not know if $\mathfrak{C}^{*}=\mathfrak{C}$ is provable in ZFC. In view of the results we have presented, it is natural to ask the following.

Problem 4.1. Is it consistent that $2^{\omega_{1}} \in \mathfrak{C}^{*}$ ?

Clearly the usual product measure on $2^{\omega_{1}}$ is of type $\omega_{1}$ and thus is not countably determined, so at the moment $2^{\omega_{1}}$ seems to be a natural candidate for proving that $\mathfrak{C}^{*}$ may be different from $\mathfrak{C}$. One can ask a question going, in a sense, in the opposite direction: Is there a nonmetrizable Corson compactum $L$ which admits an embedding $C(L) \hookrightarrow C\left(2^{\omega_{1}}\right)$ ? We shall note that the answer 'no' to that question follows from $\operatorname{MA}\left(\omega_{1}\right)$.

Let us recall that $\omega_{1}$ is a caliber of a compact space $K$ if for every family $\left\{V_{\xi}\right.$ : $\left.\xi<\omega_{1}\right\}$ of nonempty open subsets of $K$ there is a point $t \in K$ belonging to $V_{\xi}$ for uncountably many $\xi$ 's. Note that $\omega_{1}$ is a caliber of the space $2^{\omega_{1}}$ simply because $2^{\omega_{1}}$ is separable (let us mention that $\omega_{1}$ is a caliber of $2^{\kappa}$ for every $\kappa$; see e.g. Todorčević 27] and the references therein). Recall also that under $\operatorname{MA}\left(\omega_{1}\right)$ the cardinal number $\omega_{1}$ is a caliber of Radon measures in the sense that every uncountable family of sets of positive measure has an uncountable subfamily with nonempty intersection; see Džamonja and Plebanek [5] for a survey on calibers of measures and measure algebras.

Theorem 4.2. Suppose that $K$ and $L$ are compact spaces such that $C(K)$ embeds into $C(L)$. Assuming $M A\left(\omega_{1}\right)$, if $\omega_{1}$ is a caliber of $L$, then it is also a caliber of $K$.

Proof. Let us fix an isomorphic embedding $T: C(K) \rightarrow C(L)$ and consider a family $\left\{V_{\xi}: \xi<\omega_{1}\right\}$ of nonempty open subsets of $K$. For every $\xi<\omega_{1}$ choose a function $g_{\xi} \in C(K)$ such that $\left\|g_{\xi}\right\|=1$ and $g_{\xi}$ vanishes outside $V_{\xi}$. As $T$ is an embedding there is $\varepsilon>0$ such that $\left\|T g_{\xi}\right\|>\varepsilon$ for all $\xi<\omega_{1}$. Write $H_{\xi}=\left\{t \in L:\left|T g_{\xi}(t)\right|>\varepsilon\right\}$. Then the sets $H_{\xi}, \xi<\omega_{1}$, form an uncountable family of nonempty open subsets of $L$. Thus there is an uncountable $I \subseteq \omega_{1}$ and $t_{0} \in L$ such that $\left|T g_{\xi}\left(t_{0}\right)\right|>\varepsilon$ for $\xi \in I$. Without loss of generality we can assume that all numbers $T g_{\xi}\left(t_{0}\right)$ have the same sign; say they are all $>\varepsilon$ for $\xi \in I$. Let $\sigma=T^{*} \delta_{t_{0}} \in M(K)$. Then for $\xi \in I$ we have

$$
\sigma\left(g_{\xi}\right)=T^{*} \delta_{t_{0}}\left(g_{\xi}\right)=T g_{\xi}\left(t_{0}\right)>\varepsilon
$$

If we let $\mu$ be the positive part of the signed measure $\sigma$, then $\mu\left(V_{\xi}\right) \geq \mu\left(g_{\xi}\right)>\varepsilon$ whenever $\xi \in I$. Now it follows from $\mathrm{MA}\left(\omega_{1}\right)$ that there is an uncountable $J \subseteq I$ such that $\bigcap_{\xi \in J} V_{\xi} \neq \emptyset$, and we are done.

Corollary 4.3. Suppose that $K$ is Corson compact, $L$ is a compact space having caliber $\omega_{1}$, and $C(K) \hookrightarrow C(L)$. If $M A\left(\omega_{1}\right)$ holds, then $K$ is metrizable.

Proof. It is easy to check that if $\omega_{1}$ is a caliber of a Corson compactum $K$, then $K$ is necessarily metrizable (using the very definition of Corson compacta; cf. [1]). Therefore the assertion follows from Theorem 4.2

Corollary 4.4. Suppose that $K$ is Corson compact and $C(K) \hookrightarrow C\left(2^{\omega_{1}}\right)$. If $M A\left(\omega_{1}\right)$ holds, then $K$ is metrizable.

The last corollary is essentially known. It can be derived from a result due to Hagler [10], which, in particular, says that if $K$ is not metrizable and $C(K)$ embeds into $C\left(2^{\omega_{1}}\right)$, then $C(K)$ contains an isomorphic copy of the Banach space $l_{1}\left(\omega_{1}\right)$. 
In turn this yields that $K$ carries a measure of uncountable type (cf. Haydon [11]), which is impossible if $K \in \mathfrak{C}$ and MA $\left(\omega_{1}\right)$ holds.

We do not know if Corollary 4.4 can be proved in the usual set theory; however, the assertion of Corollary 4.3 is not true under $\mathrm{CH}$. Indeed, consider again the Kunen space $L$ mentioned at the end of section 3 and let $\mu \in P(L)$ be a measure of countable type supported by $L$. Then $C(L)$ may be treated as a closed subspace of $L_{\infty}(\mu)$, and $L_{\infty}(\mu) \equiv L_{\infty}[0,1] \simeq l_{\infty} \equiv C(\beta \omega)$.

\section{REFERENCES}

1. S. Argyros, S. Mercourakis, S. Negrepontis, Functional analytic properties of Corson-compact spaces, Studia Math. 89 (1988), 197-228. MR956239(90e:46020)

2. G. Borodulin-Nadzieja, Measures on minimally generated Boolean algebras, Topology Appl. 154 (2007), 3107-3124. MR2364639 (2009c:28008)

3. J. Bourgain, D.H. Fremlin, M. Talagrand, Pointwise compact sets of measurable functions, Amer. J. Math. 100 (1978), 845-886. MR.509077 (80b:54017)

4. J. Diestel, Sequences and series in Banach spaces, Springer, 1984. MR737004 (85i:46020)

5. M. Džamonja, G. Plebanek, Precalibre pairs of measure algebras, Topology Appl. 144 (2004), 67-94. MR2097129 (2005j:03041)

6. M. Džamonja, G. Plebanek, On Efimov spaces and Radon measures, Topology Appl. 154 (2007), 2063-2072. MR2324916 (2008j:54028)

7. M. Džamonja, G. Plebanek, Strictly positive measures on Boolean algebras, J. Sym. Logic 73 (2008), 1416-1432. MR2467227 (2010b:03077)

8. E. Medina Galego, On isomorphic classification of $C\left(\mathbf{2}^{\mathrm{m}} \oplus[0, \alpha]\right)$, Fund. Math. 204 (2009), 87-95. MR2507691

9. G. Godefroy, Compacts de Rosenthal, Pacific J. Math. 91 (1980), 293-306. MR615679 (82f:54030)

10. J. Hagler, On the structure of $S$ and $C(S)$ for $S$ dyadic, Trans. Amer. Math. Soc. 213 (1975), 415-428. MR0388062 (52:8899)

11. R. Haydon, On dual $L^{1}$-spaces and injective bidual Banach spaces, Israel J. Math. 31 (1978), 142-152. MR.516250 (80e:46013)

12. O. Kalenda, Valdivia compact spaces in topology and Banach space theory, Extracta Math. 15 (2000), 1-85. MR1792980 (2001k:46024)

13. P. Koszmider, The interplay between compact spaces and the Banach spaces of their continuous functions, in: Open Problems in Topology II, E. Pearl (ed.), Elsevier, 2007. MR 2367385 (2008j:54001)

14. K. Kunen, A compact L-space under CH, Topology Appl. 12 (1981), 283-287. MR623736 (82h:54065)

15. K. Kunen, J. van Mill, Measures on Corson compact spaces, Fund. Math. 147 (1995), 61-72. MR:1330107 (96c:54040)

16. W. Marciszewski, Rosenthal compacta, in: Encyclopedia of General Topology, Elsevier, 2004, 142-144. MR2049453 (2005d:54001)

17. W. Marciszewski, G. Plebanek, On measures on Rosenthal compacta, preprint (2010).

18. S. Mercourakis, Some remarks on countably determined measures and uniform distribution of sequences, Monats. Math. 121 (1996), 79-101. MR.1375642 (97j:28029)

19. S. Negrepontis, Banach spaces and topology, in: Open problems in topology, J. van Mill and G.M. Reed (eds.), Chapter 23, North-Holland Publishing Co., Amsterdam, 1990. MR 1078636 (92c:54001)

20. G. Plebanek, On some properties of Banach spaces of continuous functions, Seminaire d'Initiation à l'Analyse (G. Choquet et al.), 31 (1991/92), Université Paris VI.

21. G. Plebanek, Nonseparable Radon measures and small compact spaces, Fund. Math. 153 (1997), 25-40. MR 1450994 (98m:28025)

22. G. Plebanek, Approximating Radon measures on first-countable compact spaces, Colloq. Math. 86 (2000), 15-23. MR.1799884 (2002a:28014)

23. G. Plebanek, Convex Corson compacta and Radon measures, Fund. Math. 175 (2002), 143154. MR1969632(2004d:28027) 
24. R. Pol, Note on the spaces of regular probability measures whose topology is determined by countable subsets, Pacific J. Math. 100 (1982), 185-201. MR661448 (83g:54024)

25. M. Talagrand, Pettis integral and measure theory. Mem. Amer. Math. Soc. 307 (1984). MR 756174 (86j:46042)

26. S. Todorčević, Compact sets of the first Baire class, J. Amer. Math. Soc. 12 (1999), 1179-1212. MR 1685782 (2000d:54028)

27. S. Todorčević, Chain-condition methods in topology, Topology Appl. 101 (2000), 45-82. MR 1730899 (2001a:54055)

Institute of Mathematics, University of Warsaw, ul. Banacha 2, 02-097 Warszawa, POLAND

E-mail address: wmarcisz@mimuw.edu.pl

Mathematical Institute, University of Wroceaw, Pl. Grunwaldzki 2/4, 50-384 Wroceaw, Poland

E-mail address: grzes@math.uni.wroc.pl 